\title{
Eigenvectors of Open Bazhanov-Stroganov Quantum Chain
}

\author{
Nikolai IORGOV
}

Bogolyubov Institute for Theoretical Physics, $14 b$ Metrolohichna Str., Kyiv, 03143 Ukraine E-mail: iorgov@bitp.kiev.ua

Received November 29, 2005, in final form January 30, 2006; Published online February 04, 2006

Original article is available at http://www.emis.de/journals/SIGMA/2006/Paper019/

\begin{abstract}
In this contribution we give an explicit formula for the eigenvectors of Hamiltonians of open Bazhanov-Stroganov quantum chain. The Hamiltonians of this quantum chain is defined by the generation polynomial $A_{n}(\lambda)$ which is upper-left matrix element of monodromy matrix built from the cyclic $L$-operators. The formulas for the eigenvectors are derived using iterative procedure by Kharchev and Lebedev and given in terms of $w_{p}(s)$-function which is a root of unity analogue of $\Gamma_{q}$-function.
\end{abstract}

Key words: quantum integrable systems; Bazhanov-Stroganov quantum chain

2000 Mathematics Subject Classification: 81R12; 81R50

\section{Introduction}

In the papers $[1,2]$ it was observed that the six-vertex $R$-matrix at root of unity intertwines not only the six-vertex $L$-operators, but also some other $L$-operators (which are called cyclic $L$-operators). These $L$-operators define $n$-particle Bazhanov-Stroganov quantum chain (BSQC) by the standard procedure in quantum inverse scattering method: the product of $L$-operators defines the monodromy matrix

$$
T_{n}(\lambda)=L_{1}(\lambda) L_{2}(\lambda) \cdots L_{n}(\lambda)=\left(\begin{array}{cc}
A_{n}(\lambda) & B_{n}(\lambda) \\
C_{n}(\lambda) & D_{n}(\lambda)
\end{array}\right),
$$

which in turn provides us with commuting set of operators - Hamiltonians of quantum chain. It was observed by Baxter that the so-called "Inverse SOS" model discovered by him [3] is equivalent to Bazhanov-Stroganov quantum chain. Thus the same model (which is called the $\tau_{2}$-model) has two formulations: the formulation as face model by Baxter and the formulation as quantum chain (or vertex model) by Bazhanov and Stroganov. The connection between sixvertex model, $\tau_{2}$-model and chiral Potts model gave a possibility to formulate the system of functional relations $[1,4]$ for transfer-matrices of these models. This system provides the main tool for derivation the free energy [5] and the order parameter [6] for chiral Potts model.

The goal of this contribution is to find common eigenvectors of the set of commuting Hamiltomians $\boldsymbol{H}_{k}, k=1,2, \ldots, n$, of open $n$-particle BSQC. These Hamiltonians are defined by the coefficients of the $A_{n}(\lambda)$ given by (1):

$$
A_{n}(\lambda)=1+\lambda \boldsymbol{H}_{1}+\lambda^{2} \boldsymbol{H}_{2}+\cdots+\lambda^{n} \boldsymbol{H}_{n} .
$$

The main idea how to find the eigenvectors is to use iterative procedure. Namely we build the eigenvectors of $A_{n}(\lambda)$ using the eigenvectors of $A_{n-1}(\lambda)$ which is the generation function of Hamiltonians for open $(n-1)$-particle BSQC. This procedure in essential is an adaptation 
of iterative procedure by Kharchev and Lebedev [7] for quantum Toda chain. The mentioned idea has origin in the paper by Sklyanin [8], where he used separated variables of subsystems to construct the separated variables of the whole system. The models where the iterative procedure was realized are relativistic Toda chain [9], Toda chain with boundary interaction [10], periodic Bazhanov-Stroganov model [11]. It is worth to note that these models do not admit algebraic Bethe ansatz procedure because in the case of generic parameters these models do not possess "highest weight vectors". The method used in this contribution is an evolution of the method of separated variables (or functional Bethe ansatz method).

At the end of the introduction we would like to mention that the Bazhanov-Stroganov model at special values of parameters reduces to the relativistic Toda chain at a root of unity. Also it is worth noting direct (not through the chiral Potts model!) relation [12] between lattice formulation of the model at $N=2$ and Ising model. This relation gave a possibility to find the eigenvalues [12] and the eigenvectors [13] of the transfer-matrix by means of auxiliary grassmann field technique.

\section{Bazhanov-Stroganov quantum chain}

Let $\omega=e^{2 \pi \mathrm{i} / N}, N \geq 2$. For each particle $k, k=1,2, \ldots, n$, of Bazhanov-Stroganov quantum chain (BSQC) with $n$ particles there corresponds $N$-dimensional linear space (quantum space) $\mathcal{V}_{k}$ with the basis $|\gamma\rangle_{k}, \gamma \in \mathbb{Z}_{N}$, and a pair of operators $\left\{\boldsymbol{u}_{k}, \boldsymbol{v}_{k}\right\}$ acting on $\mathcal{V}_{k}$ by the formulas:

$$
\boldsymbol{v}_{k}|\gamma\rangle_{k}=\omega^{\gamma}|\gamma\rangle_{k}, \quad \boldsymbol{u}_{k}|\gamma\rangle_{k}=|\gamma-1\rangle_{k} .
$$

The space of quantum states of BSQC with $n$ particles is $\mathcal{V}=\mathcal{V}_{1} \otimes \mathcal{V}_{2} \otimes \cdots \otimes \mathcal{V}_{n}$. We extend the action of operators $\left\{\boldsymbol{u}_{k}, \boldsymbol{v}_{k}\right\}$ to $\mathcal{V}$ defining this action to be identical on $\mathcal{V}_{s}, s \neq k$. Thus we have the following commutation relations

$$
\boldsymbol{u}_{j} \boldsymbol{u}_{k}=\boldsymbol{u}_{k} \boldsymbol{u}_{j}, \quad \boldsymbol{v}_{j} \boldsymbol{v}_{k}=\boldsymbol{v}_{k} \boldsymbol{v}_{j}, \quad \boldsymbol{u}_{j} \boldsymbol{v}_{k}=\omega^{\delta_{j, k}} \boldsymbol{v}_{k} \boldsymbol{u}_{j} .
$$

For each particle of BSQC model we put into correspondence the cyclic $L$-operator

$$
L_{k}(\lambda)=\left(\begin{array}{cc}
1+\lambda \varkappa_{k} \boldsymbol{v}_{k} & \lambda \boldsymbol{u}_{k}^{-1}\left(a_{k}-b_{k} \boldsymbol{v}_{k}\right) \\
\boldsymbol{u}_{k}\left(c_{k}-d_{k} \boldsymbol{v}_{k}\right) & \lambda a_{k} c_{k}+\boldsymbol{v}_{k} b_{k} d_{k} / \varkappa_{k}
\end{array}\right), \quad k=1,2, \ldots, n,
$$

where $\left\{a_{k}, b_{k}, c_{k}, d_{k}, \varkappa_{k}\right\}$ are (in general complex) parameters attached to $k$ th particle. In the papers $[1,2]$ it was observed that the six-vertex $R$-matrix

$$
R(\lambda, \mu)=\left(\begin{array}{cccc}
\lambda-\omega \mu & 0 & 0 & 0 \\
0 & \omega(\lambda-\mu) & \lambda(1-\omega) & 0 \\
0 & \mu(1-\omega) & \lambda-\mu & 0 \\
0 & 0 & 0 & \lambda-\omega \mu
\end{array}\right) .
$$

at root of unity $\omega=e^{2 \pi \mathrm{i} / N}$ intertwines not only the six-vertex $L$-operator, but also the cyclic $L$-operators (3):

$$
R(\lambda, \mu) L_{k}^{(1)}(\lambda) L_{k}^{(2)}(\mu)=L_{k}^{(2)}(\mu) L_{k}^{(1)}(\lambda) R(\lambda, \mu),
$$

where $L_{k}^{(1)}(\lambda)=L_{k}(\lambda) \otimes \mathbb{I}, L_{k}^{(2)}(\mu)=\mathbb{I} \otimes L_{k}(\mu)$. In fact the formulas for $L$-operators and $R$ matrix given in this contribution are close to formulas from the paper by Tarasov [14]. Original formulas given in [1] and [2] are a bit different (but equivalent). The monodromy matrix for the BSQC with $n$ particles is defined as

$$
T_{n}(\lambda)=L_{1}(\lambda) L_{2}(\lambda) \cdots L_{n}(\lambda)=\left(\begin{array}{cc}
A_{n}(\lambda) & B_{n}(\lambda) \\
C_{n}(\lambda) & D_{n}(\lambda)
\end{array}\right)
$$


and satisfies the same intertwining relation

$$
R(\lambda, \mu) T_{n}^{(1)}(\lambda) T_{n}^{(2)}(\mu)=T_{n}^{(2)}(\mu) T_{n}^{(1)}(\lambda) R(\lambda, \mu) .
$$

The intertwining relation (5) gives $\left[A_{n}(\lambda), A_{n}(\nu)\right]=0$. Therefore $A_{n}(\lambda)$ is the generating function for the commuting set of operators $\boldsymbol{H}_{1}, \ldots, \boldsymbol{H}_{n}$ :

$$
A_{n}(\lambda)=1+\lambda \boldsymbol{H}_{1}+\lambda^{2} \boldsymbol{H}_{2}+\cdots+\lambda^{n} \boldsymbol{H}_{n} .
$$

We interpret these operators $\boldsymbol{H}_{1}, \ldots, \boldsymbol{H}_{n}$ as Hamiltonians of the open BSQC. The simplest Hamiltonians are

$$
\boldsymbol{H}_{1}=\sum_{k=1}^{n} \varkappa_{k} \boldsymbol{v}_{k}+\sum_{1 \leq l<k \leq n} \boldsymbol{u}_{l}^{-1}\left(a_{l}-b_{l} \boldsymbol{v}_{l}\right) \prod_{s=l+1}^{k-1} \frac{b_{s} d_{s}}{\varkappa_{s}} \boldsymbol{v}_{s} \cdot \boldsymbol{u}_{k}\left(c_{k}-d_{k} \boldsymbol{v}_{k}\right), \quad \boldsymbol{H}_{n}=\prod_{k=1}^{n} \varkappa_{k} \boldsymbol{v}_{k} .
$$

At $b_{k}=0$ and $c_{k}=0$, the BSQC model reduces to Relativistic Toda Chain (RTC) at root of unity. The corresponding $L$-operators are

$$
L_{k}^{\mathrm{RTC}}(\lambda)=\left(\begin{array}{cc}
1+\lambda \varkappa_{k} \boldsymbol{v}_{k} & \lambda a_{k} \boldsymbol{u}_{k}^{-1} \\
-d_{k} \boldsymbol{u}_{k} \boldsymbol{v}_{k} & 0
\end{array}\right), \quad k=1,2, \ldots, n .
$$

As in BSQC model $A_{n}^{\mathrm{RTC}}(\lambda)$ is the generating function for the commuting set of operators $\boldsymbol{H}_{1}^{\mathrm{RTC}}, \ldots, \boldsymbol{H}_{n}^{\mathrm{RTC}}$. The simplest Hamiltonians for RTC are

$$
\boldsymbol{H}_{1}^{\mathrm{RTC}}=\sum_{k=1}^{n} \varkappa_{k} \boldsymbol{v}_{k}-\sum_{1 \leq l \leq n-1} a_{l} d_{l+1} \boldsymbol{u}_{l}^{-1} \boldsymbol{u}_{l+1} \boldsymbol{v}_{l+1}, \quad \boldsymbol{H}_{n}^{\mathrm{RTC}}=\prod_{k=1}^{n} \varkappa_{k} \boldsymbol{v}_{k} .
$$

Note that these operators $\boldsymbol{H}_{1}^{\mathrm{RTC}}, \ldots, \boldsymbol{H}_{n}^{\mathrm{RTC}}$ are the Hamiltonians of the RTC with open boundary condition and association with the standard operators of momenta $\boldsymbol{p}_{k}$ and positions $\boldsymbol{q}_{k}$ roughly speaking (up to constants) is $\boldsymbol{v}_{k}=\exp \boldsymbol{p}_{k}$ and $\boldsymbol{u}_{k}=\exp \boldsymbol{q}_{k}$. Then $\boldsymbol{H}_{n}^{\mathrm{RTC}}$ is the exponent of the total momentum of RTC and $\boldsymbol{H}_{1}^{\mathrm{RTC}}$ is the Hamiltonian of relativistic analogue of usual Toda chain.

\section{Eigenvalues and associated amplitudes}

In this section we give a procedure of obtaining the eigenvalues for open $n$-particle BSQC Hamiltonians $\boldsymbol{H}_{k}, k=1,2, \ldots, n$, or equivalently for $A_{n}(\lambda)$.

In the case of $a_{k}=b_{k}=c_{k}=d_{k}=0, k=1,2, \ldots, n$, we have $A_{n}(\lambda)=\prod_{k=1}^{n}\left(1+\varkappa_{k} \boldsymbol{v}_{k}\right)$.

We interpret the corresponding Hamiltonians as free (without interaction between particles) Hamiltonians. Due to (2) the standard basis vectors $\left|\gamma_{1}, \gamma_{2}, \ldots, \gamma_{n}\right\rangle=\left|\gamma_{1}\right\rangle_{1} \otimes\left|\gamma_{2}\right\rangle_{2} \otimes \cdots \otimes\left|\gamma_{n}\right\rangle_{n} \in$ $\mathcal{V}$ are eigenvectors of $A_{n}(\lambda)$ :

$$
A_{n}(\lambda)\left|\gamma_{1}, \gamma_{2}, \ldots, \gamma_{n}\right\rangle=\prod_{k=1}^{n}\left(1+\varkappa_{k} \omega^{\gamma_{k}}\right)\left|\gamma_{1}, \gamma_{2}, \ldots, \gamma_{n}\right\rangle
$$

We claim that in the general case the spectrum of $A_{n}(\lambda)$ has the form as in the case of non-interacting particles but with modified amplitudes $\varkappa_{n, k}$ :

$$
A_{n}(\lambda)\left|\gamma_{1}, \gamma_{2}, \ldots, \gamma_{n}\right\rangle=\prod_{k=1}^{n}\left(1+\varkappa_{n, k} \omega^{\gamma_{k}} \lambda\right)\left|\gamma_{1}, \gamma_{2}, \ldots, \gamma_{n}\right\rangle, \quad \gamma_{k} \in \mathbb{Z}_{N}
$$


The corresponding eigenvectors $\left|\gamma_{1}, \gamma_{2}, \ldots, \gamma_{n}\right\rangle$ are not standard basis vectors of course. To obtain their coordinates in the standard basis we will use an iterative procedure as was promised in the introduction. We start from the eigenvectors of open 1-particle BSQC. Then we construct eigenvectors of open 2-particle BSQC by addition in an appropriate way one more particle. And so on. In parallel with this procedure we have an iterative procedure of obtaining the amplitudes:

$$
\left(\varkappa_{11}:=\varkappa_{1}\right) \stackrel{+2^{\text {nd }}}{\longrightarrow} \stackrel{\text { particle }}{\longrightarrow}\left(\varkappa_{21}, \varkappa_{22}\right) \stackrel{+3^{\text {rd }}}{\longrightarrow} \stackrel{\text { particle }}{\longrightarrow} \ldots \stackrel{+n^{\text {th }} \text { particle }}{\longrightarrow}\left(\varkappa_{n 1}, \ldots, \varkappa_{n n}\right) .
$$

Now we will describe the procedure how to find these amplitudes $\varkappa_{m, s}, m=1,2, \ldots, n, s=$ $1,2, \ldots, m$. We will need the variables

$$
x_{m^{\prime}, s^{\prime}}^{m, s}=\frac{\varkappa_{m, s}}{\varkappa_{m^{\prime}, s^{\prime}}}, \quad x_{m}=\frac{c_{m}}{d_{m}}, \quad x_{m, s}=\frac{c_{m} \varkappa_{m}}{d_{m} \varkappa_{m, s}}, \quad \tilde{x}_{m, s}=\frac{b_{m} \varkappa_{m, s}}{a_{m} \varkappa_{m}},
$$

and variables $y_{m^{\prime}, s^{\prime}}^{m, s}, y_{m}, y_{m, s}, \tilde{y}_{m, s}$. The latter are defined (up to a root of 1, which will be fixed later) by condition that points $p_{m^{\prime}, s^{\prime}}^{m, s}=\left(x_{m^{\prime}, s^{\prime}}^{m, s}, y_{m^{\prime}, s^{\prime}}^{m, s}\right), p_{m}=\left(x_{m}, y_{m}\right), p_{m, s}=\left(x_{m, s}, y_{m, s}\right), \tilde{p}=$ $\left(\tilde{x}_{m, s}, \tilde{y}_{m, s}\right)$ belong to Fermat curve $x^{N}+y^{N}=1$. First, we define $\varkappa_{1,1}:=\varkappa_{1}$. If we constructed all the above variables for $m-1$ particles, we define the variables $\varkappa_{m, 1}, \varkappa_{m, 2}, \ldots, \varkappa_{m, m}$ by the equations

$$
\begin{aligned}
& \varkappa_{m, 1} \varkappa_{m, 2} \cdots \varkappa_{m, m}=\varkappa_{m-1,1} \varkappa_{m-1,2} \cdots \varkappa_{m-1, m-1} \varkappa_{m}, \\
& \frac{\varkappa_{m}}{a_{m-1} d_{m}} \frac{y_{m-1}}{y_{m} y_{m-1, l} \tilde{y}_{m-1, l}} \prod_{s \neq l} \frac{y_{m-1, l}^{m-1, s}}{y_{m-1, s}^{m-1, l}} \frac{\prod_{k=1}^{m} y_{m, k}^{m-1, l}}{\prod_{s=1}^{m-2} y_{m-1, l}^{m-2, s}}=1, \quad l=1,2, \ldots, m-1 .
\end{aligned}
$$

We would like to mention that this iterative procedure has a similarity to iterative procedures in $[15,16]$. To solve these equations we first take $N$-th power of them. It gives us system of linear equations

$$
\begin{aligned}
& \varkappa_{m, 1}^{N} \varkappa_{m, 2}^{N} \cdots \varkappa_{m, m}^{N}=\varkappa_{m-1,1}^{N} \varkappa_{m-1,2}^{N} \cdots \varkappa_{m-1, m-1}^{N} \varkappa_{m}^{N}, \\
& \frac{\varkappa_{m-1, l}^{N}}{a_{m-1}^{N} d_{m}^{N}} \frac{y_{m-1}^{N}}{y_{m}^{N} y_{m-1, l}^{N} \tilde{y}_{m-1, l}^{N}} \frac{\prod_{k=1}^{m}\left(1-\varkappa_{m, k}^{N} / \varkappa_{m-1, l}^{N}\right)}{\prod_{s=1}^{m-2}\left(1-\varkappa_{m-2, s}^{N} / \varkappa_{m-1, l}^{N}\right)}=1, \quad l=1,2, \ldots, m-1 .
\end{aligned}
$$

with respect to elementary symmetric polynomials in variables $\left\{\varkappa_{m, 1}^{N}, \ldots, \varkappa_{m, m}^{N}\right\}$. Solving equation with coefficients being the values of the mentioned symmetric polynomials we obtain the values of $\left\{\varkappa_{m, 1}^{N}, \ldots, \varkappa_{m, m}^{N}\right\}$. The variables $\left\{\varkappa_{m, 1}, \ldots, \varkappa_{m, m}\right\}$ can be found up to $N$-th roots of 1 . We fix their phases in a way to satisfy (7) and (8).

To compare these formulas with the formulas for eigenvalues proposed by Tarasov [14] we consider polynomials $\mathcal{A}_{m}\left(\lambda^{N}\right)$ with zeroes $\epsilon / \varkappa_{m, s}^{N}, s=1,2, \ldots, m$, where $\epsilon=(-1)^{N}$ :

$$
\mathcal{A}_{m}\left(\lambda^{N}\right)=\prod_{s=1}^{m}\left(1-\epsilon \varkappa_{m, s}^{N} \lambda^{N}\right), \quad m \geq 2 ; \quad \mathcal{A}_{1}\left(\lambda^{N}\right)=1-\epsilon \varkappa_{1}^{N} \lambda^{N} ; \quad \mathcal{A}_{0}\left(\lambda^{N}\right)=1 .
$$

Then the relations (9) and (10) can be rewritten compactly as recursion relation for $\mathcal{A}_{m}\left(\lambda^{N}\right)$, $m \geq 2$ :

$$
\mathcal{A}_{m}\left(\lambda^{N}\right)=\left(\left(1-\epsilon \varkappa_{m}^{N} \lambda^{N}\right)+\frac{c_{m}^{N}-d_{m}^{N}}{c_{m-1}^{N}-d_{m-1}^{N}}\left(\frac{b_{m-1}^{N} d_{m-1}^{N}}{\varkappa_{m-1}^{N}}-\epsilon \lambda^{N} a_{m-1}^{N} c_{m-1}^{N}\right)\right) \mathcal{A}_{m-1}\left(\lambda^{N}\right)
$$




$$
+\frac{c_{m}^{N}-d_{m}^{N}}{c_{m-1}^{N}-d_{m-1}^{N}} \frac{\left(b_{m-1}^{N}-\epsilon \lambda^{N} a_{m-1}^{N} \varkappa_{m-1}^{N}\right)\left(\epsilon \lambda^{N} c_{m-1}^{N} \varkappa_{m-1}^{N}-d_{m-1}^{N}\right)}{\varkappa_{m-1}^{N}} \mathcal{A}_{m-2}\left(\lambda^{N}\right) .
$$

Indeed, the relation (9) follows from the relation for coefficients in (12) at $\left(\lambda^{N}\right)^{m}$. If we fix sequentially $\lambda^{N}=\epsilon / \varkappa_{m-1, l}^{N}, l=1,2, \ldots, m-1$, (that is by the zeroes of $\mathcal{A}_{m-1}\left(\lambda^{N}\right)$ ) we obtain the relations $(10)$.

This recursion relation for $\mathcal{A}_{m}\left(\lambda^{N}\right)$ can be obtained by means of averaged $L$-operators [14]. Using

$$
\mathcal{L}_{m}\left(\lambda^{N}\right)=\left(\begin{array}{cc}
1-\epsilon \varkappa_{m}^{N} \lambda^{N} & -\epsilon \lambda^{N}\left(a_{m}^{N}-b_{m}^{N}\right) \\
c_{m}^{N}-d_{m}^{N} & b_{m}^{N} d_{m}^{N} / \varkappa_{m}^{N}-\epsilon \lambda^{N} a_{m}^{N} c_{m}^{N}
\end{array}\right)
$$

we define polynomials $\mathcal{A}_{m}\left(\lambda^{N}\right), \mathcal{B}_{m}\left(\lambda^{N}\right), \mathcal{C}_{m}\left(\lambda^{N}\right)$ and $\mathcal{D}_{m}\left(\lambda^{N}\right)$ by

$$
\left(\begin{array}{cc}
\mathcal{A}_{m}\left(\lambda^{N}\right) & \mathcal{B}_{m}\left(\lambda^{N}\right) \\
\mathcal{C}_{m}\left(\lambda^{N}\right) & \mathcal{D}_{m}\left(\lambda^{N}\right)
\end{array}\right)=\mathcal{L}_{1}\left(\lambda^{N}\right) \mathcal{L}_{2}\left(\lambda^{N}\right) \cdots \mathcal{L}_{m}\left(\lambda^{N}\right)
$$

In particular, we have

$$
\begin{aligned}
& \mathcal{A}_{m}\left(\lambda^{N}\right)=\left(1-\epsilon \varkappa_{m}^{N} \lambda^{N}\right) \mathcal{A}_{m-1}\left(\lambda^{N}\right)+\left(c_{m}^{N}-d_{m}^{N}\right) \mathcal{B}_{m-1}\left(\lambda^{N}\right), \\
& \mathcal{B}_{m}\left(\lambda^{N}\right)=-\epsilon \lambda^{N}\left(a_{m}^{N}-b_{m}^{N}\right) \mathcal{A}_{m-1}\left(\lambda^{N}\right)+\left(b_{m}^{N} d_{m}^{N} / \varkappa_{m}^{N}-\epsilon \lambda^{N} a_{m}^{N} c_{m}^{N}\right) \mathcal{B}_{m-1}\left(\lambda^{N}\right) .
\end{aligned}
$$

Excluding $\mathcal{B}_{m-1}\left(\lambda^{N}\right)$ from these two relations we get

$$
\mathcal{B}_{m}\left(\lambda^{N}\right)=\frac{b_{m}^{N} d_{m}^{N} / \varkappa_{m}^{N}-\epsilon \lambda^{N} a_{m}^{N} c_{m}^{N}}{c_{m}^{N}-d_{m}^{N}} \mathcal{A}_{m}\left(\lambda^{N}\right)-\frac{\operatorname{det} \mathcal{L}_{m}\left(\lambda^{N}\right)}{c_{m}^{N}-d_{m}^{N}} \mathcal{A}_{m-1}\left(\lambda^{N}\right) .
$$

Substituting the right-hand side of this equation with $m$ replaced by $m-1$ instead of $\mathcal{B}_{m-1}\left(\lambda^{N}\right)$ in (14) we get (12). Therefore two formulas (12) and (13) for $\mathcal{A}_{m}\left(\lambda^{N}\right)$ are equivalent. Summarizing, in order to find amplitudes $\varkappa_{m, s}, s=1, \ldots, m$, for some $m$, we have to find $\mathcal{A}_{m}\left(\lambda^{N}\right)$ using (12) or (13). Then solving equation $\mathcal{A}_{m}\left(\lambda^{N}\right)=0$ of $m$ th degree with respect to $\lambda^{N}$ and taking into account (11) we can find $\varkappa_{m, s}^{N}, s=1, \ldots, m$. This gives us the set $\varkappa_{m, s}$ up to $N$ th roots of 1 . At last step, we have to fix their values in a way to satisfy (7) and (8).

It seems to the author that the equation $\mathcal{A}_{m}\left(\lambda^{N}\right)=0$ can not be solved explicitly in the case of generic parameters. In the next section, the solution for the homogeneous RTC is given explicitly. The author does not know other interesting special cases of parameters which admit explicit solution for the spectrum of $A_{m}(\lambda)$. As shown in [11], it is possible to give an explicit solution for the spectrum of $B_{m}(\lambda)$ in the homogeneous case of $m$-particle Bazhanov-Stroganov quantum chain.

\section{Amplitudes for the homogeneous Relativistic Toda Chain}

In this section we sketch the method described in [15] of obtaining the amplitudes for the homogeneous RTC: $a_{k}=a, b_{k}=0, c_{k}=0, d_{k}=d, \varkappa_{k}=\varkappa$. In this case the amplitudes $\varkappa_{m, s}$, $s=1, \ldots, m$, can be expressed in terms of solutions of some quadratic equation. Since

$$
\mathcal{L}_{k}^{\mathrm{RTC}}\left(\lambda^{N}\right)=\mathcal{L}^{\mathrm{RTC}}\left(\lambda^{N}\right)=\left(\begin{array}{cc}
1-\epsilon \varkappa^{N} \lambda^{N} & -\epsilon a^{N} \lambda^{N} \\
-d^{N} & 0
\end{array}\right),
$$

we obtain

$$
\left(\begin{array}{ll}
\mathcal{A}_{m}\left(\lambda^{N}\right) & \mathcal{B}_{m}\left(\lambda^{N}\right) \\
\mathcal{C}_{m}\left(\lambda^{N}\right) & \mathcal{D}_{m}\left(\lambda^{N}\right)
\end{array}\right)=\left(\mathcal{L}^{\mathrm{RTC}}\left(\lambda^{N}\right)\right)^{m}
$$


Applying the fact that $2 \times 2$ matrix $M$ with eigenvalues $\mu_{+}$and $\mu_{-}$satisfies

$$
\boldsymbol{M}^{m}=\frac{\mu_{+}^{m}-\mu_{-}^{m}}{\mu_{+}-\mu_{-}} \boldsymbol{M}-\frac{\mu_{+}^{m} \mu_{-}-\mu_{-}^{m} \mu_{+}}{\mu_{+}-\mu_{-}} \mathbf{1}
$$

for matrix $\mathcal{L}^{\mathrm{RTC}}\left(\lambda^{N}\right)$ we obtain

$$
\mathcal{A}_{m}\left(\lambda^{N}\right)=\left(1-\epsilon \varkappa^{N} \lambda^{N}\right) \frac{x_{+}^{m}-x_{-}^{m}}{x_{+}-x_{-}}-\frac{x_{+}^{m} x_{-}-x_{-}^{m} x_{+}}{x_{+}-x_{-}},
$$

where $x_{+}\left(\lambda^{N}\right)$ and $x_{-}\left(\lambda^{N}\right)$ are eigenvalues of $\mathcal{L}\left(\lambda^{N}\right)$. These eigenvalues are roots of characteristic polynomial $x^{2}-\tau\left(\lambda^{N}\right) x+\delta\left(\lambda^{N}\right)=0$ :

$$
x_{ \pm}=\frac{1}{2}\left(\tau \pm \sqrt{\tau^{2}-4 \delta}\right)
$$

where, using (15),

$$
\begin{aligned}
& \tau\left(\lambda^{N}\right)=\operatorname{tr} \mathcal{L}^{\mathrm{RTC}}\left(\lambda^{N}\right)=x_{1}+x_{2}=1-\epsilon \varkappa^{N} \lambda^{N}, \\
& \delta\left(\lambda^{N}\right)=\operatorname{det} \mathcal{L}^{\mathrm{RTC}}\left(\lambda^{N}\right)=x_{1} x_{2}=-\epsilon a^{N} d^{N} \lambda^{N} .
\end{aligned}
$$

Taking into account (17) we rewrite (16) as

$$
\mathcal{A}_{m}\left(\lambda^{N}\right)=\frac{x_{+}^{m+1}-x_{-}^{m+1}}{x_{+}-x_{-}} .
$$

Introducing the variable $\phi$ by $x_{+} / x_{-}=e^{\mathrm{i} \phi}$ we find that roots of $\mathcal{A}_{m}$ correspond to roots $\phi_{m, s}$ of $e^{\mathrm{i}(m+1) \phi}=1$ (without $\phi=0$ ) that is

$$
\phi_{m, s}=2 \pi s /(m+1), \quad s=1,2, \ldots, m .
$$

Now we need to find an explicit relation between $\lambda^{N}$ and $\phi$. We have

$$
\tau+\sqrt{\tau^{2}-4 \delta}=e^{\mathrm{i} \phi}\left(\tau-\sqrt{\tau^{2}-4 \delta}\right) .
$$

Therefore

$$
\tau^{2}=4 \delta \cos ^{2} \frac{\phi}{2}
$$

Taking into account (17) and (18) we consider (20) as quadratic equation with respect to $\lambda^{N}$ :

$$
\lambda^{2 N} \varkappa^{2 N}+2 \epsilon \lambda^{N}\left(a^{N} d^{N}+a^{N} d^{N} \cos \phi-\varkappa^{N}\right)+1=0 .
$$

The solution $\lambda^{N}(\phi)$ of this equation describes the relation between the variables $\lambda^{N}$ and $\phi$. Therefore we can translate the zeroes (19) of $\mathcal{A}_{m}\left(\lambda^{N}(\phi)\right)$ in terms of variable $\phi$ to zeroes $\lambda^{N}\left(\phi_{m, s}\right)$ in terms of $\lambda^{N}$. Finally, taking into account (11) we find

$$
\varkappa_{m, s}^{N}=\epsilon / \lambda^{N}\left(\phi_{m, s}\right), \quad s=1,2, \ldots, m \text {. }
$$




\section{$5 \quad$ Eigenvectors and eigenvalues}

In order to give explicit formulas for the eigenvectors of $A_{n}(\lambda)$ we remind the definition (see for example [17]) of $w_{p}(s)$ which is an analogue of $\Gamma_{q}$-function at root of unity. For any point $p=(x, y)$ of Fermat curve $x^{N}+y^{N}=1$, we define $w_{p}(s), s \in \mathbb{Z}_{N}$, by

$$
\frac{w_{p}(s)}{w_{p}(s-1)}=\frac{y}{1-x \omega^{s}}, \quad w_{p}(0)=1
$$

The function $w_{p}(s)$ is cyclic: $w_{p}(s+N)=w_{p}(s)$.

We will use the notation $\left|\gamma_{n}\right\rangle \in \mathcal{V}_{1} \otimes \cdots \otimes \mathcal{V}_{n}$ for eigenvectors of the operator $A_{n}(\lambda)$ of the BSQC with $n$ particles. These eigenvectors are labeled by $n$ parameters $\gamma_{n, s} \in \mathbb{Z}_{N}, s=$ $1,2, \ldots, n$, collected into a vector

$$
\gamma_{n}=\left(\gamma_{n, 1}, \ldots, \gamma_{n, n}\right) \in\left(\mathbb{Z}_{N}\right)^{n} .
$$

The following theorem gives a procedure of obtaining the eigenvectors $\left|\gamma_{n}\right\rangle$ of $A_{n}(\lambda)$ from the eigenvectors $\left|\gamma_{n-1}\right\rangle \in \mathcal{V}_{1} \otimes \cdots \otimes \mathcal{V}_{n-1}$ of $A_{n-1}(\lambda)$ and basis vectors $\left|\gamma_{n}\right\rangle_{n} \in \mathcal{V}_{n}$. To find the formula for $\left|\gamma_{n-1}\right\rangle$ we can use the same theorem and so on. At the last step we need the eigenvectors of 1-particle quantum chain.

From (3) and (4), it is easy to see that the vectors $\left|\gamma_{1,1}\right\rangle_{1} \in \mathcal{V}_{1}, \gamma_{1,1} \in \mathbb{Z}_{N}$, are eigenvectors for $A_{1}(\lambda)$ :

$$
A_{1}(\lambda)\left|\gamma_{1,1}\right\rangle_{1}=\left(1+\varkappa_{1,1} \omega^{\gamma_{1,1}}\right)\left|\gamma_{1,1}\right\rangle_{1},
$$

where $\varkappa_{1,1}=\varkappa_{1}$.

In what follows, the vector $\gamma_{n}^{ \pm k}$ means the vector $\gamma_{n}$ in which $\gamma_{n, k}$ is replaced by $\gamma_{n, k} \pm 1$.

Theorem 1. The vector $\left|\gamma_{n}\right\rangle=\left|\gamma_{n 1}, \ldots, \gamma_{n n}\right\rangle$

$$
\left|\gamma_{n}\right\rangle=\sum_{\gamma_{n-1} \in\left(\mathbb{Z}_{N}\right)^{n-1}} Q\left(\gamma_{n-1} \mid \gamma_{n}\right)\left|\gamma_{n-1}\right\rangle \otimes\left|\sigma_{n}\right\rangle_{n}
$$

satisfies

$$
\begin{aligned}
A_{n}(\lambda)\left|\gamma_{n}\right\rangle= & \prod_{k=1}^{n}\left(1+\varkappa_{n, k} \omega^{\gamma_{n, k}} \lambda\right)\left|\gamma_{n}\right\rangle=\prod_{k=1}^{n}\left(1-\lambda / \lambda_{n, k}\right)\left|\gamma_{n}\right\rangle \\
B_{n}\left(\lambda_{n, k}\right)\left|\gamma_{n}\right\rangle= & \frac{a_{n} \lambda_{n, k}}{y_{n}}\left(1-x_{n, k} \omega^{-\gamma_{n, k}-1}\right)\left(1-\tilde{x}_{n, k} \omega^{\gamma_{n, k}}\right)\left(\prod_{s=1}^{n-1} y_{n, k}^{n-1, s}\right)\left|\gamma_{n}^{+k}\right\rangle, \\
B_{n}(\lambda)\left|\gamma_{n}\right\rangle= & \lambda \frac{a_{n}}{y_{n}} \sum_{k=1}^{n}\left(\prod_{s \neq k} \frac{\lambda-\lambda_{n, s}}{\lambda_{n, k}-\lambda_{n, s}}\right) \\
& \times\left(1-x_{n, k} \omega^{-\gamma_{n, k}-1}\right)\left(1-\tilde{x}_{n, k} \omega^{\gamma_{n, k}}\right)\left(\prod_{l=1}^{n-1} y_{n, k}^{n-1, l}\right)\left|\gamma_{n}^{+k}\right\rangle, \quad n>1, \\
B_{1}(\lambda)\left|\gamma_{1}\right\rangle= & \lambda a_{1}\left(1-\tilde{x}_{1,1} \omega^{\gamma_{1,1}}\right)\left|\gamma_{1}^{+1}\right\rangle,
\end{aligned}
$$

if $\left|\gamma_{n-1}\right\rangle=\left|\gamma_{n-1,1}, \ldots, \gamma_{n-1, n-1}\right\rangle \in \mathcal{V}_{1} \otimes \cdots \otimes \mathcal{V}_{n-1}$ satisfies the same relations with $n$ replaced by $n-1$. In the above formulas we used

$$
Q\left(\gamma_{n-1} \mid \gamma_{n}\right)=\frac{\omega^{\gamma_{n-1,1}+\cdots+\gamma_{n-1, n-1}} \prod_{l=1}^{n-1} \prod_{k=1}^{n} w_{p_{n, k}^{n-1, l}}\left(\gamma_{n-1, l}-\gamma_{n, k}\right)}{w_{p_{n}}\left(-\sigma_{n}-1\right) \prod_{\substack{j, l=1 \\(j \neq l)}}^{n-1} w_{p_{n-1, l}^{n-1, j}}\left(\gamma_{n-1, j}-\gamma_{n-1, l}\right)} \cdot \frac{\prod_{l=1}^{n-1} w_{p_{n-1, l}}\left(-\gamma_{n-1, l}-1\right)}{\prod_{l=1}^{n-1} w_{\tilde{p}_{n-1, l}}\left(\gamma_{n-1, l}-1\right)},
$$




$$
\begin{aligned}
& Q\left(\gamma_{1} \mid \gamma_{2}\right)=\frac{\omega^{\gamma_{1,1}} w_{p_{2,1}^{1,1}}\left(\gamma_{1,1}-\gamma_{2,1}\right) w_{p_{2,2}^{1,1}}\left(\gamma_{1,1}-\gamma_{2,2}\right)}{w_{p_{2}}\left(\gamma_{1,1}-\gamma_{2,1}-\gamma_{2,2}-1\right) w_{\tilde{p}_{1,1}}\left(\gamma_{1,1}-1\right)} \\
& \lambda_{m, s}=-\omega^{-\gamma_{m, s}} / \varkappa_{m, s}, \quad \sigma_{n}\left(\gamma_{n-1}, \gamma_{n}\right) \equiv \sigma_{n}=\sum_{k=1}^{n} \gamma_{n, k}-\sum_{l=1}^{n-1} \gamma_{n-1, l} .
\end{aligned}
$$

Proof. We suppose that the formulas (23) and (25) with $n$ replaced by $n-1$ are proved. To prove the action formulas (23) and (25) we use the recurrent relations

$$
\begin{aligned}
& A_{n}(\lambda)=A_{n-1}(\lambda)\left(1+\lambda \varkappa_{n} \boldsymbol{v}_{n}\right)+B_{n-1}(\lambda) \boldsymbol{u}_{n}\left(c_{n}-d_{n} \boldsymbol{v}_{n}\right), \\
& B_{n}(\lambda)=A_{n-1}(\lambda) \lambda \boldsymbol{u}_{n}^{-1}\left(a_{n}-b_{n} \boldsymbol{v}_{n}\right)+B_{n-1}(\lambda)\left(\lambda a_{n} c_{n}+\frac{b_{n} d_{n}}{\varkappa_{n}} \boldsymbol{v}_{n}\right)
\end{aligned}
$$

which follow from (4).

The action formula for $A_{n}(\lambda)$ : To prove the action formula (23) we act by both sides of (28) on (22) and use the formulas (23) and (25) with $n$ replaced by $n-1$. After shifting in an appropriate way the variables of summation $\gamma_{n-1}$ we reduce the problem to verification of relation

$$
\begin{aligned}
\left(\prod_{k=1}^{n}(1\right. & \left.\left.-\lambda / \lambda_{n, k}\right)-\prod_{l=1}^{n-1}\left(1-\lambda / \lambda_{n-1, l}\right)\left(1+\lambda \varkappa_{n} \omega^{\sigma_{n}}\right)\right) Q\left(\gamma_{n-1} \mid \gamma_{n}\right) \\
= & \lambda \frac{a_{n-1}}{y_{n-1}} \sum_{l=1}^{n-1}\left(\prod_{\substack{s=1 \\
s \neq l}}^{n-1} \frac{\lambda-\lambda_{n-1, s}}{\omega \lambda_{n-1, l}-\lambda_{n-1, s}}\right)\left(1-x_{n-1, l} \omega^{-\gamma_{n-1, l}}\right)\left(1-\tilde{x}_{n-1, l} \omega^{\gamma_{n-1, l}-1}\right) \\
& \times\left(\prod_{s=1}^{n-2} y_{n-1, l}^{n-2, s}\right)\left(c_{n}-d_{n} \omega^{\sigma_{n}+1}\right) Q\left(\gamma_{n-1}^{-l} \mid \gamma_{n}\right) .
\end{aligned}
$$

Using

$$
\begin{gathered}
\frac{Q\left(\gamma_{n-1}^{-l} \mid \gamma_{n}\right)}{Q\left(\gamma_{n-1} \mid \gamma_{n}\right)} \frac{a_{n-1}}{y_{n-1}}\left(1-x_{n-1, l} \omega^{-\gamma_{n-1, l}}\right)\left(1-\tilde{x}_{n-1, l} \omega^{\gamma_{n-1, l}-1}\right)\left(\prod_{s=1}^{n-2} y_{n-1, l}^{n-2, s}\right) \\
=\frac{\varkappa_{n}}{\omega d_{n}} \frac{\prod_{k=1}^{n}\left(1-\frac{\lambda_{n, k}}{\lambda_{n-1, l}}\right)}{1-x_{n} \omega^{-\sigma_{n}-1}} \prod_{s \neq l} \frac{1-\omega \frac{\lambda_{n-1, l}}{\lambda_{n-1, s}}}{1-\frac{\lambda_{n-1, s}}{\lambda_{n-1, l}}}
\end{gathered}
$$

which follows directly from (27) and (8), we rewrite (30) as

$$
\begin{aligned}
& \prod_{k=1}^{n}\left(1-\lambda / \lambda_{n, k}\right)-\prod_{l=1}^{n-1}\left(1-\lambda / \lambda_{n-1, l}\right)\left(1+\lambda \varkappa_{n} \omega^{\sigma_{n}}\right) \\
& \quad=\lambda \sum_{l=1}^{n-1}\left(\prod_{\substack{s=1 \\
s \neq l}}^{n-1} \frac{\lambda-\lambda_{n-1, s}}{\lambda_{n-1, l}-\lambda_{n-1, s}}\right) \frac{-\varkappa_{n} \omega^{\sigma_{n}}}{\lambda_{n-1, l}} \frac{\prod_{k=1}^{n} \lambda_{n, k}}{\prod_{s=1}^{n-1} \lambda_{n-1, s}} \prod_{k=1}^{n}\left(1-\lambda_{n-1, l} / \lambda_{n, k}\right) .
\end{aligned}
$$

Taking into account

$$
-\varkappa_{n} \omega^{\sigma_{n}} \frac{\prod_{k=1}^{n} \lambda_{n, k}}{\prod_{s=1}^{n-1} \lambda_{n-1, s}}=1,
$$


which follows from (7), we obtain finally

$$
\begin{aligned}
\prod_{k=1}^{n}\left(1-\lambda / \lambda_{n, k}\right)-\prod_{l=1}^{n-1}\left(1-\lambda / \lambda_{n-1, l}\right)\left(1+\lambda \varkappa_{n} \omega^{\sigma_{n}}\right) \\
\quad=\lambda \sum_{l=1}^{n-1}\left(\prod_{\substack{s=1 \\
s \neq l}}^{n-1} \frac{\lambda-\lambda_{n-1, s}}{\lambda_{n-1, l}-\lambda_{n-1, s}}\right) \frac{1}{\lambda_{n-1, l}} \prod_{k=1}^{n}\left(1-\lambda_{n-1, l} / \lambda_{n, k}\right) .
\end{aligned}
$$

To verify this equality we note that both sides are polynomials in $\lambda$ of degree $n-1$ (not $n$ due to (31)) without free term. Therefore it is sufficient to verify this relation at $n-1$ different values of $\lambda$. Taking these values to be $\lambda=\lambda_{n-1, l}, l=1,2, \ldots, n-1$, we easily prove the relation. Thus we proved (23).

The action formula for $B_{n}\left(\lambda_{n, k}\right)$ : Next we show the validity of (24). The action formulas for $B_{1}(\lambda)$ and $B_{2}(\lambda)$ can be verified in a direct way. Thus we suppose $n>2$.

Excluding $B_{n-1}(\lambda)$ from (28) and substituting it into (29) we get

$$
\begin{aligned}
\boldsymbol{u}_{n}\left(c_{n}-d_{n} \boldsymbol{v}_{n}\right) B_{n}(\lambda) & =\left(\lambda a_{n} c_{n}+\omega \frac{b_{n} d_{n}}{\varkappa_{n}} \boldsymbol{v}_{n}\right) A_{n}(\lambda) \\
& -\omega \lambda a_{n} d_{n} \boldsymbol{v}_{n}\left(1+\frac{\lambda c_{n} \varkappa_{n}}{\omega d_{n}}\right)\left(1+\frac{b_{n}}{\lambda a_{n} \varkappa_{n}}\right) A_{n-1}(\lambda) .
\end{aligned}
$$

Let us apply (32) to $\left|\gamma_{n}\right\rangle$ for $\lambda=\lambda_{n, k}=-\omega^{-\gamma_{n, k}} / \varkappa_{n, k}$, i.e. at the zeros of eigenvalue of $A_{n}(\lambda)$. This gives, by virtue of the definitions (6) of $x_{m, s}$ and $\tilde{x}_{m, s}$ :

$$
\begin{gathered}
\boldsymbol{u}_{n}\left(c_{n}-d_{n} \boldsymbol{v}_{n}\right) B_{n}\left(\lambda_{n, k}\right)\left|\gamma_{n}\right\rangle=-\lambda_{n, k} \omega a_{n} d_{n}\left(1-x_{n, k} \omega^{-\gamma_{n, k}-1}\right)\left(1-\tilde{x}_{n, k} \omega^{\gamma_{n, k}}\right) \\
\times \sum_{\gamma_{n-1} \in\left(\mathbb{Z}_{N}\right)^{n-1}} \omega^{\sigma_{n}} Q\left(\gamma_{n-1} \mid \gamma_{n}\right) A_{n-1}\left(\lambda_{n, k}\right)\left|\gamma_{n-1}\right\rangle \otimes\left|\sigma_{n}\right\rangle .
\end{gathered}
$$

From (23) we know how to apply $A_{n-1}$ to $\left|\boldsymbol{\gamma}_{n-1}\right\rangle$ :

$$
A_{n-1}\left(\lambda_{n, k}\right)\left|\gamma_{n-1}\right\rangle=\prod_{s=1}^{n-1}\left(1-\frac{\varkappa_{n-1, s}}{\varkappa_{n, k}} \omega^{\gamma_{n, k}-\gamma_{n-1, s}}\right)\left|\gamma_{n-1}\right\rangle .
$$

Using (2) we find the action of the inverse of the operator $\boldsymbol{u}_{n}\left(c_{n}-d_{n} \boldsymbol{v}_{n}\right)$ on $\left|\sigma_{n}\right\rangle_{n}$ :

$$
\left(\boldsymbol{u}_{n}\left(c_{n}-d_{n} \boldsymbol{v}_{n}\right)\right)^{-1}\left|\sigma_{n}\right\rangle_{n}=\left(c_{n}-d_{n} \omega^{\sigma_{n}+1}\right)^{-1}\left|\sigma_{n}+1\right\rangle_{n}
$$

Taking into account (27) and (21) we get

$$
Q\left(\boldsymbol{\gamma}_{n-1} \mid \boldsymbol{\gamma}_{n}\right) \frac{\omega^{\sigma_{n}} \prod_{s=1}^{n-1}\left(1-\frac{\varkappa_{n-1, s}}{\varkappa_{n, k}} \omega^{\gamma_{n, k}-\gamma_{n-1, s}}\right)}{c_{n}-d_{n} \omega^{\sigma_{n}+1}}=-\omega^{-1} Q\left(\boldsymbol{\gamma}_{n-1} \mid \boldsymbol{\gamma}_{n}^{+k}\right) \frac{\prod_{s=1}^{n-1} y_{n, k}^{n-1, s}}{d_{n} y_{n}} .
$$

Finally, using (34), (35) and (36), we reduce (33) to (24).

The action formula for $B_{n}(\lambda)$ : From (3) and (4) it is easy to find that the operator $B_{n}(\lambda) / \lambda$ is a polynomial in $\lambda$ of $(n-1)$ th order. Due to (24) we know the action formulas for $B_{n}(\lambda) / \lambda$ at the $n$ particular values of $\lambda: \lambda=\lambda_{n, k}, k=1,2, \ldots, n$. This data is enough to reconstruct the action of the polynomial $B_{n}(\lambda)$ on $\left|\gamma_{n}\right\rangle$ uniquely. Lagrange interpolation formula gives

$$
\frac{B_{n}(\lambda)}{\lambda}\left|\gamma_{n}\right\rangle=\sum_{k=1}^{n}\left(\prod_{l \neq k} \frac{\lambda-\lambda_{n, l}}{\lambda_{n, k}-\lambda_{n, l}}\right) \frac{B_{n}\left(\lambda_{n, k}\right)}{\lambda_{n, k}}\left|\gamma_{n}\right\rangle
$$

Finally using (24) we get (25). This completes the proof of the Theorem. 


\section{Discussion}

In this contribution we applied the iterative procedure of obtaining the eigenvectors for quantum integrable systems by Kharchev and Lebedev [7] (which has origin in [8] by Sklyanin) to open Bazhanov-Stroganov quantum chain. We plan to extend this result (along the line of the paper [10]) to the case of Bazhanov-Stroganov chain with integrable boundary interaction.

\section{Acknowledgements}

The author would like to acknowledge the organizers of the Sixth International Conference "Symmetry in Nonlinear Mathematical Physics" (June 20-26, Kyiv) for their nice conference. The present paper is the written version of the talk delivered by the author at this conference. The author is thankful to Professors G. von Gehlen, S. Pakuliak and V. Shadura for collaboration in obtaining the results presented in this contribution. The research presented here is partially supported by INTAS (grant No.03-51-3350) and by the French-Ukrainian project "Dnipro".

[1] Bazhanov V.V., Stroganov Yu.G., Chiral Potts model as a descendant of the six-vertex model, J. Statist. Phys., 1990, V.59, 799-817.

[2] Korepanov I.G., Hidden symmetries in the 6-vertex model, Chelyabinsk Polytechnical Inst., VINITI No. 1472-V87, 1987 (in Russian).

Korepanov I.G., Hidden symmetries in the 6-vertex model of statistical physics, Zap. Nauchn. Sem. S.-Peterburg. Otdel. Mat. Inst. Steklov. (POMI), 1994, V.215, 163-177 (English transl.: J. Math. Sci. (New York), 1997, V.85, 1661-1670); hep-th/9410066.

[3] Baxter R.J., Superintegrable chiral Potts model: thermodynamic properties, an "inverse" model, and a simple associated Hamiltonian, J. Statist. Phys., 1989, V.57, 1-39.

[4] Baxter R.J., Bazhanov V.V., Perk J.H.H., Functional relations for the transfer matrices of the chiral Potts model, Internat. J. Modern Phys. B, 1990, V.4, 803-869.

[5] Baxter R.J., Chiral Potts model: eigenvalues of the transfer matrix, Phys. Lett. A, 1990, V.146, $110-114$.

[6] Baxter R.J., Derivation of the order parameter of the chiral Potts model, Phys. Rev. Lett., 2005, V.94, 130602, 3 pages; cond-mat/0501227.

[7] Kharchev S., Lebedev D., Eigenfunctions of $G L(N, \mathbb{R})$ Toda chain: the Mellin-Barnes representation, JETP Lett., 2000, V.71, 235-238; hep-th/0004065.

[8] Sklyanin E.K., Quantum inverse scattering method. Selected topics, in Nankai Lectures in Mathematical Physics "Quantum Group and Quantum Integrable Systems", Editor Mo-Lin Ge, Singapore, World Scientific, 1992, 63-97.

[9] Kharchev S., Lebedev D., Semenov-Tian-Shansky M., Unitary representations of $U_{q}(\operatorname{sl}(2, \mathbb{R}))$, the modular double, and the multiparticle $q$-deformed Toda chains, Comm. Math. Phys., 2002, V.225, N 3, 573-609; hep-th/0102180.

[10] Iorgov N., Shadura V., Wave functions of the Toda chain with boundary interaction, Theoret. and Math. Phys., 2005, V.142, N 2, 289-305; nonlin.SI/0411002.

[11] von Gehlen G., Iorgov N., Pakuliak S., Shadura V., Baxter-Bazhanov-Stoganov model: separation of variables and Baxter equation, in preparation.

[12] Bugrij A.I., Iorgov N.Z., Shadura V.N., Alternative method of calculating the eigenvalues of the transfer matrix of the $\tau_{2}$ model for $N=2$, JETP Lett., 2005, V.82, 311-315.

[13] Lisovyy O., Transfer matrix eigenvectors of the Baxter-Bazhanov-Stroganov $\tau_{2}$-model for $N=2, J$. Phys. A: Math. Gen., 2006, V.39, to appear; nlin.SI/0512026.

[14] Tarasov V.O., Cyclic monodromy matrices for the $R$-matrix of the six-vertex model and the chiral Potts model with fixed spin boundary conditions, Internat. J. Modern Phys. A Suppl., 1992, V.7, 963-975.

[15] Pakuliak S., Sergeev S., Quantum relativistic Toda chain at root of unity: isospectrality, modified $Q$-operator and functional Bethe ansatz, Int. J. Math. Math. Sci., 2002, V.31, 513-554; nlin.SI/0205037.

[16] von Gehlen G., Pakuliak S., Sergeev S., The Bazhanov-Stroganov model from 3D approach, J. Phys. A: Math. Gen., 2005, V.38, 7269-7298; nlin.SI/0505019.

[17] Bazhanov V.V., Baxter R.J., Star-triangle relation for a three dimensional model, J. Statist. Phys., 1993, V.71, 839-864; hep-th/9212050. 